\title{
EDSAC Replica Project
}

\author{
Andrew Herbert and David Hartley \\ The National Museum of Computing, UK \\ david.hartley@tnmoc.org
}

\begin{abstract}
EDSAC (The Electronic Delay Storage Automatic Calculator) was the world's first practical stored program electronic computer. The goal of the EDSAC Replica Project is to build a functional replica of the University of Cambridge EDSAC Computer as it was in 1949, and for the replica to be a working demonstration at the UK National Museum of Computing.
\end{abstract}

Keywords: EDSAC, replica, functional.

\section{EDSAC Background}

The Electronic Delay Storage Automatic Calculator (EDSAC) was the world's first practical stored program electronic computer. Running its first program on $6^{\text {th }}$ May 1949 EDSAC provided a scientific computing service to Cambridge University ahead of any similar provision elsewhere. To its users EDSAC represented a thousand fold increment in computing power over the mechanical calculators that preceded it and contributed to three Cambridge Nobel Prizes by enabling faster and larger scale computations than had hitherto been practical.

EDSAC was designed by Maurice Wilkes (later Professor Sir Maurice Wilkes) of the Cambridge University Mathematical Laboratory in 1947. At the instigation of Professor Douglas Hartree, an eminent Cambridge mathematician, Wilkes attended the famous Moore School Lectures at the University of Pennsylvania in the summer of 1946 at which the American computer pioneers presented experience with machines such as the ENIAC and perhaps more importantly, Von Neumann presented a paper on the design of the EDVAC that first crystallised the principles of operation for modern stored program computers. Greatly influenced by EDVAC, Wilkes sketched a design for EDSAC while travelling home on the Queen Mary and on arrival back in Cambridge in September 1946 he assembled a team of engineers led by W. Renwick to start construction of a machine.

Events moved quickly after the first successful program ran on $6^{\text {th }}$ May 1949: the machine was completed and a conference took place the following month by which time the computer was already in regular service. EDSAC continued in use for nearly 10 years until replaced by its successor, EDSAC 2. Throughout its life EDSAC underwent continual improvement and by the end of its life, the size of the store had doubled from 512 to 102418 bit words, and additional instructions, an index register and magnetic tape storage facilities had been added. 


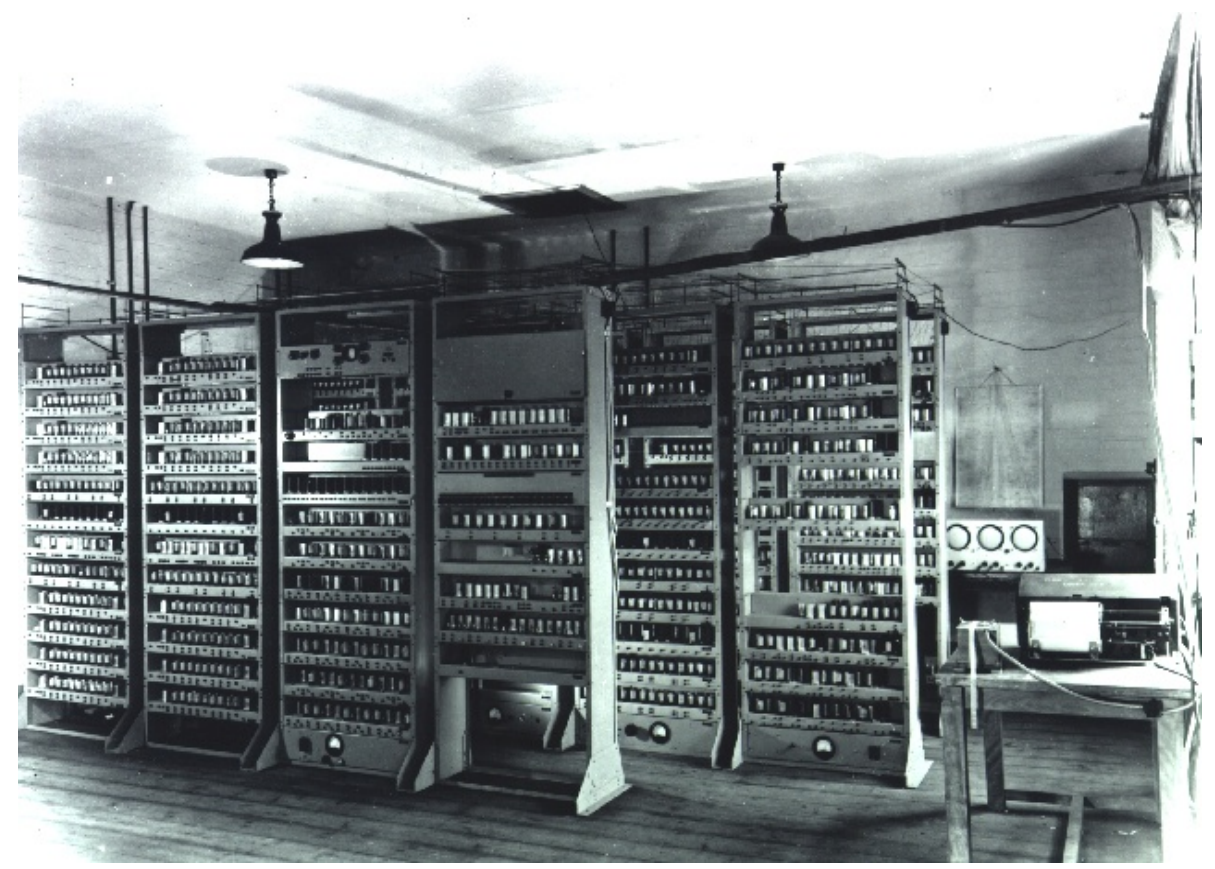

Fig. 1. EDSAC, May 1949

By the standards of the time, EDSAC ran reliably throughout its life, typically delivering 35 hours of computation a week. Outside of normal hours it often ran unattended by engineers, under the control of authorized users. This can be attributed to the conservative nature of Wilkes' design. The central clock ran at $500 \mathrm{KHz}$, allowing generous margins for internal synchronisation and the architecture was serial, reducing the number of circuits to be constructed from large and expensive thermionic valves.

EDSAC was fondly remembered by its users, many of whom attended a $50^{\text {th }}$ anniversary conference at Cambridge in 1999. Sadly little of the machine survives in physical form and the documentary record in the Cambridge University library is fragmentary. Over the last decade, with the death of Wilkes and most of those who worked with him, knowledge of this important machine in the history of computing is rapidly fading away.

\section{Project Aims}

The goal of the EDSAC Replica Project is to build a functional replica of EDSAC as it was in 1949. By functional replica we are expressing the intention to build a machine that runs programs as EDSAC would have done in 1949, which appears physically as similar as possible to the appearance of EDSAC in photographs, is consistent with the surviving documentation and where we have to re-invent parts of the machine, we do so using appropriate historical circuit designs and assembly 
techniques. We plan to exhibit the EDSAC replica at the UK National Museum of Computing at Bletchley Park.

Our aims for the replica project are that it should:

- $\quad$ Provide a tangible demonstration of the achievements of the Cambridge pioneers.

- $\quad$ Celebrate the achievements of British scientific and technological contributions to the early development of computing.

- Assemble archive material relating to this historic machine and capture the knowledge of the few remaining pioneers who developed and used it.

- Build as authentic a replica as possible taking into account the availability of components and materials and modern safety standards.

- Undertake the work within the spirit of the designers and technology of the time.

- $\quad$ Produce a working artefact of exceptional educational value to students and the general public.

- $\quad$ Be an exemplar of British engineering and encourage new students to take up computing and engineering.

- Demonstrate the working machine to the public as often as is practical once it is built and commissioned at The National Museum of Computing.

- $\quad$ Be constructed and equipped with sufficient spares to enable the machine to run for the next 25 years.

- Train a new generation of volunteers, unfamiliar with 1940s valve technology, to build, run, demonstrate and maintain the replica for the foreseeable future.

\section{$3 \quad$ Project Organization}

The second author of this paper (David Hartley) with the encouragement of Dr Hermann Hauser, a well-known Cambridge technology entrepreneur originally conceived the project. Led by Hartley, the University of Cambridge, the British Computer Society and the Hauser-Raspe Foundation jointly set up The EDSAC Replica Project as a registered UK charity. Initial funding came from The HauserRaspe Foundation, Google and a private individual who have, at the time of writing, generously contributed half of the estimated project cost of $£ 250,000$.

Volunteers are undertaking much of the construction of the replica with some work contracted out to commercial suppliers. The first author (Andrew Herbert) is the Project Manager. Equally important in the leadership of the project is the role of Chris Burton as Chief Designer. Chris brings to the project experience of the restoration of a Ferranti Pegasus computer at the London Science Museum and the construction of a functional replica of the University of Manchester Small Scale Experimental Machine ("Baby") for the Manchester Museum of Science and Industry. The target date for completion is mid to late 2015.

Wilkes was unsentimental about the machines he designed: when EDSAC 2 was ready for use, EDSAC was unceremoniously scrapped to make room for it. Just a few 
pieces were sent to museums: 3 examples of the most common type of EDSAC chassis and 3 short mercury delay line tubes.

A number of accessories such as hand punches for correcting paper tape also survive but that is all. Fortunately there is a reasonably good photographic record: Wilkes had official photographs made from glass-plate negatives and these remain in the Cambridge University Library. His own personal photograph album has been donated by his family to the Cambridge University Computer Laboratory to put alongside other personal photographs collected at the time of the EDSAC'99 conference. Importantly a $195116 \mathrm{~mm}$ colour film made in the Mathematical Laboratory (the former name of the present Computer Laboratory) showing the operation of the machine survives. However most of the images are undated and it can be seen that significant changes were made to the machine throughout its life and therefore care has to be taken in using them to settle questions about EDSAC's appearance in 1949.

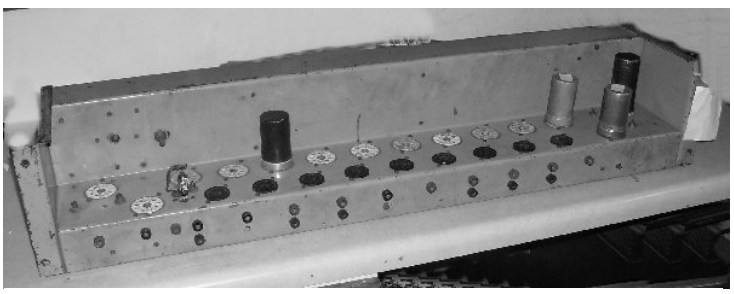

The documentary record is on the surface rather more complete. A key document is The EDSAC Report deposited by Wilkes in the University Library. This gives a good overview of the architecture of Fig. 2. Surviving EDSAC Chassis the machine and its logical design. There are hints at some of the circuit elements used but no description of the physical layout. Moreover it is unclear whether the EDSAC Report is a design specification or a write-up of the completed machine, or perhaps something in between, so it too must be used with caution.

We then have academic papers written about EDSAC. Mostly these are at the architectural level and focus on how EDSAC was programmed - another of the EDSAC innovations was the simplicity and convenience of its programming system. A few papers give more detailed engineering information, particularly about the mercury delay lines used for the main store.

Then there are Wilkes' memoirs and his speeches on EDSAC to various historical

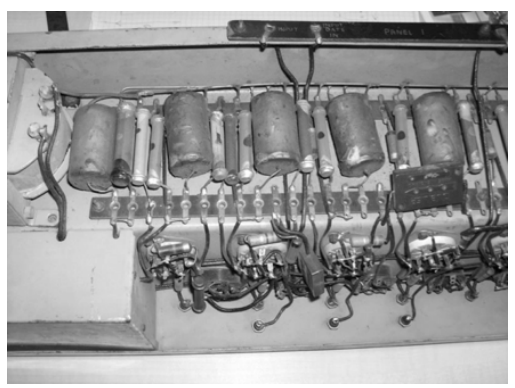

Fig. 3. Chassis Underside societies and computer associations, but these tend to speak more to the context in which EDSAC was conceived and used than in giving technical detail.

Finally we have some surviving notebooks from the project team and "EDSAC Operating Memoranda" and "EDSAC Programming Bulletins" issued to users of EDSAC that shed some light on technical detail. Early documents on the Lyons LEO I computer have also proved useful: the design of LEO was based on EDSAC 
and comments in LEO papers about "improvements" relative to EDSAC help us to reverse engineer the original design.

In summary, the project has enough information to know the principles of how EDSAC worked and was used, but lacks most of the detail required to construct an exact replica. The essential project task is to bridge this gap.

\section{EDSAC Physical Design}

Physically EDSAC consisted of three rows of open racks, each row comprising four, three and five racks respectively and each rack holding up to 14 shelves containing electronics. (Wilkes called the shelves "panels", and in the project we follow more modern terminology and call them "chassis"; our mechanical engineering subcontractor calls them "shelves"). The total footprint of the racks is about $5 \mathrm{~m}^{2}$ and they are approximately $2.5 \mathrm{~m}$ high. In total there are 142 chassis.

In addition to the racks, further floor space was taken up by wooden "coffins" containing the mercury delay line tanks used for the main store and registers. The coffins provided a level of temperature stability and were approximately $2 \mathrm{~m}$ long. The store was later moved to a thermostatically controlled oven.

The operator controlled the machine from a simple panel with a Reset and Start button mounted on one of the racks: a paper tape reader for input and teleprinter for output were placed on a wooden table. A separate engineer's desk accommodated a threeoscilloscope unit that allowed the contents of selected memory tubes to be inspected. (Later in the life of EDSAC all these elements moved to a single operator's desk).

EDSAC was powered from a motor generator unit in a separate room: the electrical consumption is estimated at $15 \mathrm{KW}$.

In terms of building a replica, the racks and chassis are relatively straightforward to reconstruct as we have a sample chassis and several photographs from which we can estimate leading dimensions. Working with a local engineering company in Cambridge (Teversham Engineering Ltd) we have CAD models for chassis and racks. In contrast to the original, where making a chassis would probably have taken an engineer most of a day in the Mathematical Laboratory's workshops, using their Computer Aided manufacturing systems, Teversham Engineering can cut, punch, drill and fold chassis in a matter of minutes.

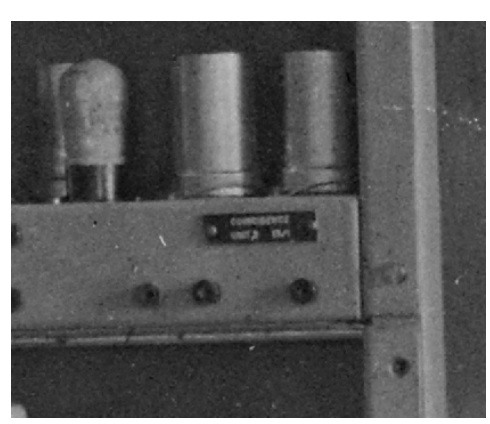

Fig. 4. Illegible Chassis Label

The chassis model is a generic template as different chassis had different punching depending upon the circuits it contained. This is where difficulties arise - we do not have a photograph of every chassis and over the lifetime of EDSAC some chassis were moved, or changed and new functions introduced. To further frustrate us, we know each chassis had an identifying engraved label, and while we can see these on the photographs, the resolution is insufficient to read them, even with modern image enhancement techniques. 
We therefore have had to co-design the physical structure of the machine with the logical design and the circuit design. From logical design we can predict which circuit elements might have been used, and from those likely physical layouts of valves and test points on chassis: these can then be matched to photographs. We can further reasonably assume that chassis implementing related parts of the same set of logical functions (e.g., arithmetic, computer control, store addressing etc.) are grouped close together and from that make a well-informed guess as to where those chassis might have been placed in the racks if not fully visible.

In making the physical racks and chassis we have followed original dimensions but for convenience substituted modern metric fixings for the frames. On the chassis we have retained the BA fixings used on the original.

Surprisingly while the valves we need have been relatively easy to obtain from dealers, valve holders have been more difficult and top clips impossible. It is possible to get valve holders made in quantity but they are expensive: they can also be imported from the Far East but the quality is unsatisfactory. In the case of International Octal sockets we have located slightly oversize items and turned them down to standard size to fit the chassis.

Similarly we have been able to get tag strips for connecting components manufactured in bulk, a great convenience as we need at least two for every chassis. Customised tag strips are required for the connections between chassis: these are being hand made as each chassis type has a unique design. Fortunately a suitable punch, tags and blank paxolin strips could be obtained from the same source as the manufactured strips.

Another area requiring custom manufacture has been the provision of electromagnetic delay line inductances. These consist of wound coils on a Tufnol former wired to capacitors on a thick wire frame. Through the British Vintage Wireless Society we have been able to get the coils wound to our specification. The manufacture of formers and wiring up is being undertaken by project volunteers.

An area in which we have chosen to compromise is with passive components such as resistors and capacitors. Modern components are generally much smaller than their equivalents from the 1940s and painted differently. While old components can be found, they have often drifted away from their nominal value and the potential for them failing in use is high. We have therefore decided to use modern components for reasons of reliability. Where possible we look for modern components of a suitably large size (for example by choosing resistors of a higher power rating than necessary) so that the visual appearance is suitable. Further, through our relationship with the British Vintage Wireless Society we have located some suppliers of modern components to old designs. Given the scale of EDSAC we have decided against following some of the society's practices for hand building lookalike versions of old components. To a significant degree this compromise will not impact the appearance of the replica as most of the passive components lie underneath the chassis, and the valves, for which we are using genuine parts, are the most visible and characteristic aspect. We may choose to build one or two representative chassis with correct vintage components for illustrative purposes and to emphasise that the use of more modern ones elsewhere has not compromised the integrity of the replica machine. 


\section{EDSAC Logical Design}

Recovery of the logical design of EDSAC was a key first achievement of the project. Fortunately, from the EDSAC Report and early papers about EDSAC there is a wealth of detail about the functional units within the machine and the data paths between them. The EDSAC Report also contains a number of timing diagrams

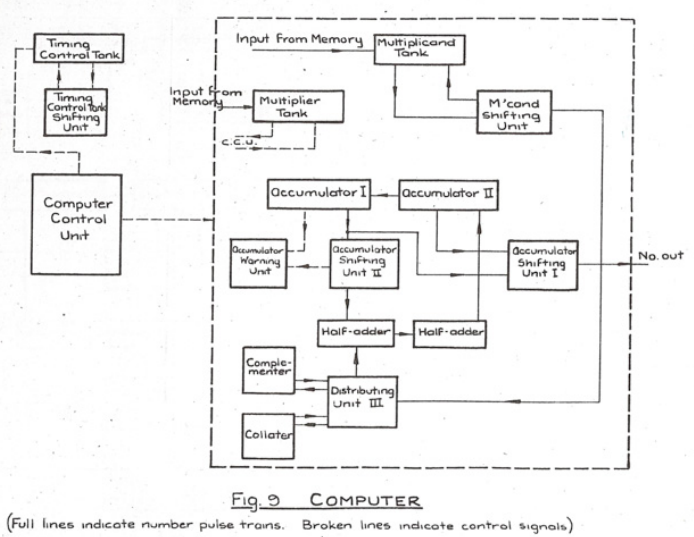

Fig. 5. A Logic Diagram from the EDSAC Report showing how signals are delayed as they pass through parts of the machine. From this information Bill Purvis has constructed an EDSAC logic simulator called ELSIE that reads in a description of the machine and simulates execution of a program. By stepwise refinement Bill was able to arrive at a design that correctly executes EDSAC programs and was consistent with the functions for which we have descriptions. A number of areas such as paper tape input, teleprinter output and initial instruction loading have been left as black-box subsystems, but most of the rest of the machine has been analysed to a sufficient level of detail to allow circuit design to start.

In early versions of ELSIE signals were represented as rectangular pulses in units of clock pulse intervals. As understanding of the logic developed and circuit elements were designed, the signal model was refined to better represent actual pulse shapes and delays to help the circuit designers determine where pulse resynchronisation and reshaping was required. In the same vein considerations of the timing of signal propagation have helped decide how close the various logical units might have been to one another.

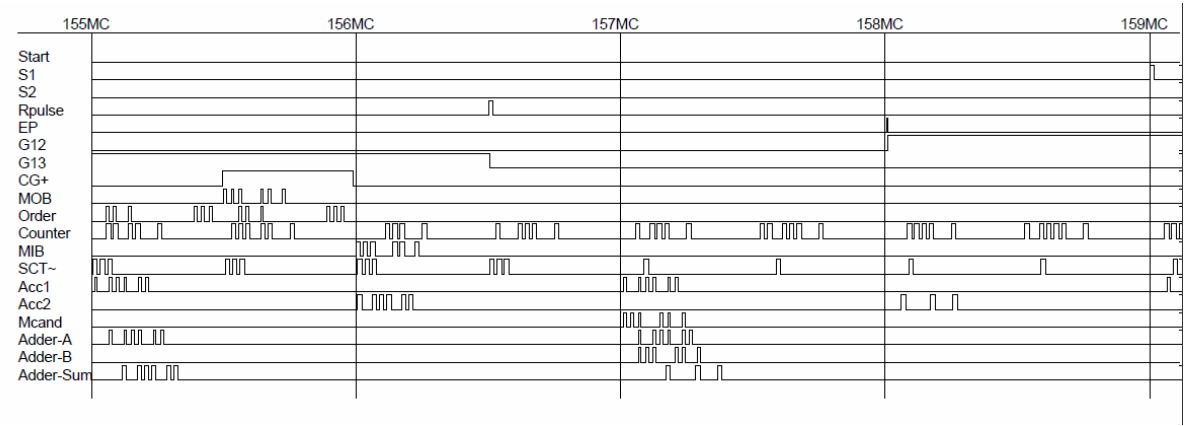

Fig. 6. Typical ELSIE Output 


\section{EDSAC Electronic Design}

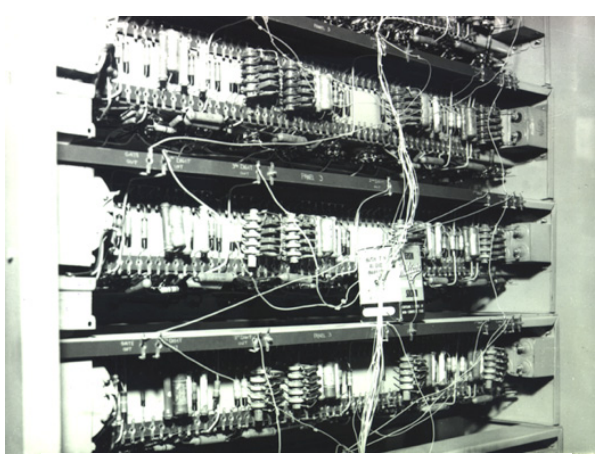

Fig. 7. EDSAC Digit Pulse Generator
Electronic design was the area where we had least to start with: the EDSAC Report shows a circuit for a flip-flop, but without any component values stated: we had some hand-drawn diagrams for the clock and digit pulse generating subsystem and just one original chassis from the storage regeneration sub-system. From various papers and notebooks we had some references to problems with specific circuits and the names of circuit elements used. We also know Wilkes had a wartime background in radar and we can look to period textbooks to understand typical circuit elements from that context that might have been used or adapted for EDSAC. From these sketchy foundations Chris Burton has established a library of standard circuits for logic gates, pulse amplifiers and flip-flops for other project volunteers to use as they design complete circuits for logical functions such as the central clocking, arithmetic unit and so forth. Where we know the physical location of the function in the original machine and have a suitable photograph we can check the physical valve layout against the electronic design, and if necessary revise the electronic design to match the physical layout. Circuit design has been further complicated by the fact EDSAC is a serial machine and A.C. coupled making circuit principles unfamiliar to those who have grown up in the transistor age with parallel data paths and D.C. coupled circuits.

From the surviving original chassis and a few close-up photographs of chassis from the early days, Chris has also been able to lay down constructional principles for how individual components should be wired together, standard values chosen for components such as stopper resistors and so forth.

It is sobering to modern eyes to realise that a simple AND gate for EDSAC takes 3 pentode and two double diode thermionic valves, so with a maximum capacity on a shelf of about 18-20 valves, each chassis is limited to 3-4 gates only. With a total of 3,000 valves therefore EDSAC is equivalent to less than 600 gates (and indeed significantly less than this given the need for valve amplifiers to drive output signals etc.). It is easy to appreciate why Wilkes and his team went for a serial rather than parallel architecture.

A recurrent challenge in the on-going work to design replica EDSAC circuits is to avoid the temptation to design a better EDSAC. The temptation comes from several sources. We know from surviving notebooks that some parts of EDSAC were temperamental: the pioneers found workarounds for such problems rather than wholesale redesign as their driving purpose was to get to a fully working machine as soon as possible. Some circuits were later replaced by improved designs. but these are outside the scope of our goal to replicate EDSAC as it was in 1949. For example we have access to more modern components, e.g., plugs and sockets for interconnecting chassis rather than soldering wires between tag strips that would be more convenient and safer. We have been ruthless to avoid such temptations with two exceptions. 
First we are putting a $0 \mathrm{~V}$ (zero volt) rail on each chassis to help with electrical safety: on the original $0 \mathrm{~V}$ rail was the chassis itself and chassis were tied together and thence to ground. This is a reversible "improvement" - we can chose to connect each such rail to chassis if we change our minds. Second, while we continue with the principle of using wiring between tag strips to interconnect chassis, we are using modern grip fasteners ("fastons") that clip tightly to the tags.

A related concern is the distribution of power and high voltage DC around the machine. The original machine had a motor generator delivering power to each rack that was distributed by soldering wires between short tag strips. We are exploring the possibility of using smaller modern power supplies on a per rack basis and modern connectors to feed power to each chassis for the obvious safety reasons. We also plan to use modern RCCBs for earth leakage and electric shock prevention. In these respects we will be thoroughly in the modern world, and in that spirit our working practices when commissioning and operating the machine will be subject to appropriate levels of health and safety management - something clearly not on the minds of the pioneers judging by some of the hazards visible in the early photographs!

For archival purposes, all of our circuit designs have been captured digitally using "DesignSpark", a circuit design and PCB layout program available from one of the major suppliers of electronic components (RSComponents). We also have a formal system of "Hardware Notes" for capturing key design decisions and principles and investigations into prototype circuits etc.

\section{$7 \quad$ EDSAC Memory}

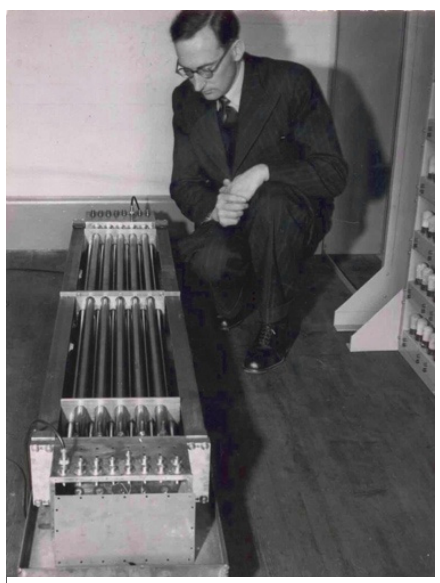

Fig. 8. Wilkes and Mercury Delay Lines
The main store of EDSAC and its internal registers were constructed using mercury delay lines. This was a technology familiar to Wilkes from his wartime radar work, and through a colleague, Tommy Gold, he had the expertise needed to make mercury delay lines for EDSAC. These delay lines present a significant challenge to the replica project. Mercury is expensive and has to be handled as a dangerous substance. The precision engineering required to manufacture the required tubes and end plates is demanding. In service, the delay lines were temperature sensitive and a source of unreliability. Moreover the mercury attacked the seals in the units that had to be replaced and the mercury itself "cleaned" every couple of years.

All these considerations make replicating a mercury delay line store impractical. During the commissioning stage we plan to build an interim store using a modern microcontroller so that, in addition to simulating delay lines, we can provide facilities to preload and inspect store. We will arrange that the interim store has the same electric signal input 
and output as the original mercury delay lines so that the store access and regeneration circuits will be as in the original, and the option to build a mercury delay line store in the future remains open.

One project member, Peter Linington, has been investigating "nickel delay lines" as an alternative to mercury delay lines. Nickel delay lines were a popular storage technology in the 1950s, bridging the gap between mercury delay lines and ferrite core store. The current plan is to use these to replace the interim microcontroller store. They are a close physical analogue to mercury delay lines in that both use a mechanical transducer to send an acoustic pulse through a medium to "store" it. Conveniently they are also of similar dimensions. While there are surviving example nickel delay lines that can be inspected and technical papers on the physics of the medium, the means of constructing them has

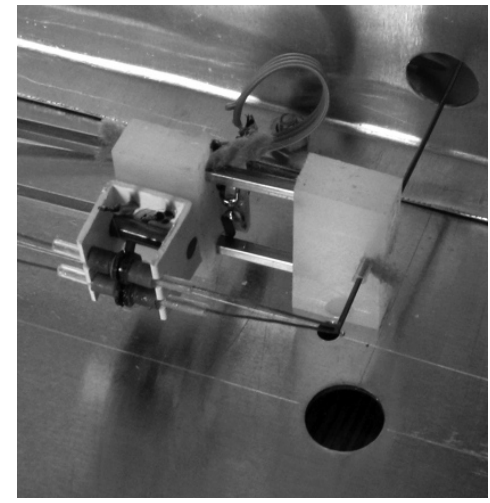

Fig. 9. Nickel Delay Line Prototype Transducer essentially been lost. Peter has been investigating techniques for annealing the wires, welding the fingers that actuate them and suitable materials to support the wires and to dampen out echoes etc. At the time of writing, he has a sample short delay line, suitable for uses as a register, which operates reliably with an error rate of 1 in $10^{11}$, i.e., roughly one bit error per day of operation. Current developments are focussed on building a long delay line suitable for use in the main store. To fit an EDSAC store "coffin" the wire needs to be coiled or folded in half. Techniques to do this are under investigation.

As a long-term ambition we are giving consideration to replicating a short delay line tank (or possibly re-commissioning one of the surviving originals) as a standalone demonstration of how mercury delay lines work.

\section{EDSAC Input-Output}

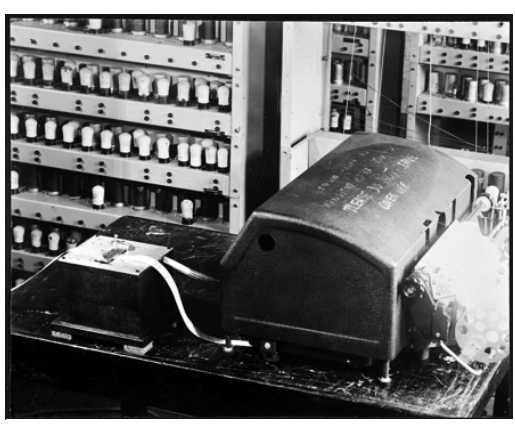

Fig. 10. EDSAC tape reader and teleprinter
The original EDSAC took input from 5-hole paper tape, read by an electro-mechanical reader constructed in the Mathematical Laboratory. From Wilkes' personal photographs we have good views of the internal mechanism and John Deane has been sketching a design for manufacturing a replica. We have from the original the sprocket wheel required to pull the tape through. Holes in the tape were mechanically sensed and relays used to capture each row of data and signal it electrically to the computer.

Output was to a teleprinter and again we 
have some photographs of the original. Bill Purvis is investigating how a Creed teleprinter from the 1950 s could be adapted to meet our needs.

Two related items of "input-output" are the means for loading the initial orders when the Reset button is pressed and the "3-oscilloscope unit" on the engineer's desk. We know from the documentation that the initial orders were wired on a bank of telephony uniselectors and injected into the main store. We also have a photograph of the unit in the original machine. Fortunately uniselectors are still obtainable, and Andrew Brown is in the early stages of designing the initial orders loading subsystem. The 3-oscilloscope unit allowed the operator to select a bank of words in the store and see a visible representation of their content on one of the scopes and the current instruction and its address on the other two: with an execution speed of around 650 instructions per second it was relatively easy to monitor program execution in this way. Currently little work has been put into understanding how this unit was connected or designed internally, but is clearly a critical part of the original that needs to be re-created.

For the initial commissioning phase of the project, and as an operating convenience thereafter, we plan to use a modern PC as a substitute for tape reader and teleprinter (and as mentioned earlier for store also). The details are yet to be worked out but our aim would be to achieve plug compatibility at the lowest level so we can easily switch out the PC and run with real peripherals.

By setting the goal that the replica match EDSAC as it was in 1949, the project team set a challenge to those interested in programming the machine. The first programs ran using "initial orders 1" but in September of that year these had been replaced by David Wheeler's "initial orders 2" which introduced facilities for subroutine linkage and relocation, making it practical to write large programs using a library of standard functions. To run the iconic first programs (table of squares, list of prime numbers) we need initial orders 1 . We knew that Wheeler had written a socalled set of "coordinating orders" that allowed initial orders 2 programs to be loaded under initial orders 1 with the coordinating orders as a prelude on their tapes. Unfortunately no description of the coordinating orders survives, and knowing of Wheeler's reputation as a masterful machine code programmer the software team doubted these could be replicated. Somewhat as an experiment we tried using initial orders 1 to load initial orders 2 to the top of store and then copy them down to the bottom end. Much to our delight this worked and so the secret of Wheeler's coordinating orders has been successfully penetrated!

\section{Current Status}

At the time of writing volunteers are wiring up chassis as follows:

1. The central Clock Pulse Generator - Peter Lawrence

2. The bank of Digit Pulse Generators - John Sanderson

3. The half adder (at the core of the "arithmetic unit") - Nigel Bennée

4. The storage access logic (tank address decoding) - John Pratt.

We have a prototype nickel delay line short tank for register memory - Peter Linington. 
Design is underway for the tape reader - John Deane, teleprinter - Bill Purvis and initial instruction loading - Andrew Brown.

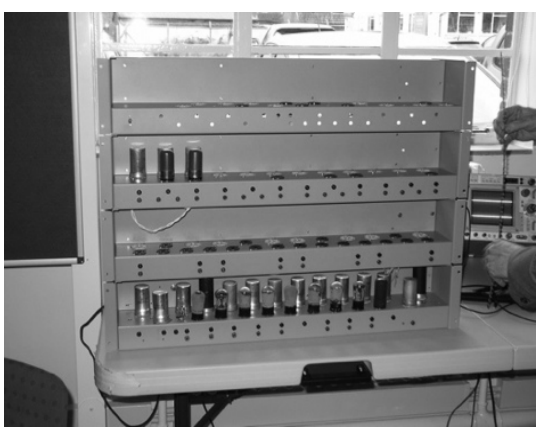

Fig. 11. Replica Chassis under Construction
In addition to the named designers above working with Chris Burton our Chief Designer, we have a further 6 or so volunteers helping with construction and most importantly, Alan Clarke who is our storekeeper and seeks out sources for all the parts required.

The aim is to have a first fully operating version of the replica at TNMOC with interim store and I/O by late summer 2015. As a first working exhibit we hope to have a rack on display with clock pulses and counting (i.e., adding) on display this summer (2013).

\section{0}

The goal of the EDSAC Replica Project is to build a functional replica of The University of Cambridge EDSAC Computer as it was when first operating in 1949 and for the replica to be a working demonstration at the UK National Museum of Computing. Little of the original EDSAC survives, but working from photographs and a fragmented documentary record, the project team have resolved many questions of physical, logical and electronic design and are, at the time of writing, constructing the first set of functional EDSAC chassis. The replica will use nickel delay lines for the main store in place of the mercury tanks of the original, but in all other respects the replica will be as close as possible to the original. The target date for project completion is 2015 .

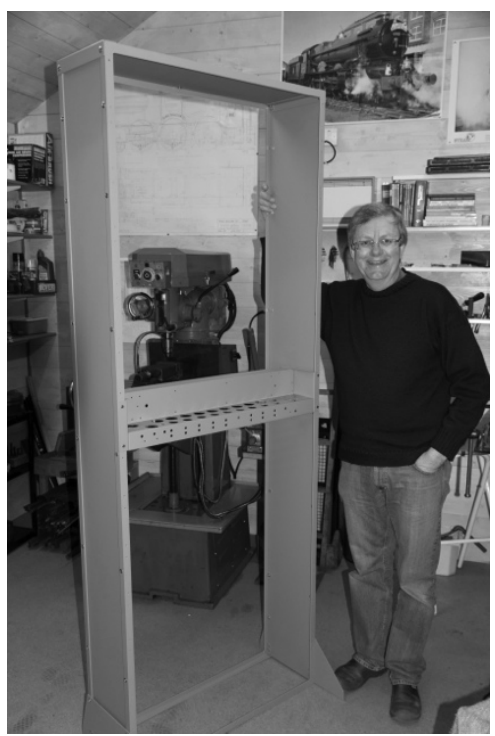

Fig. 12. 